Pacific Journal of Mathematic 


\section{ON A CRITERION FOR THE WEAKNESS OF AN IDEAL BOUNDARY COMPONENT}

\section{KôTARO OIKAWA}

1. Exhaustion. Let $F$ be an open Riemann surface. An exhaustion $\left\{F_{n}\right\}$ of $F$ is an increasing (i.e., $\bar{F}_{n} \subset F_{n+1}$ ) sequence of subregions with compact closures such that $\bigcup_{n=1}^{\infty} F_{n}=F$. We assume that $\partial F_{n}$ consists of a finite number of closed analytic curves and that each component of $F-F_{n}$ is noncompact. This is the most common definition used in the theory of open Riemann surfaces. Sometimes, however, we shall add the restriction that each component of $\partial F_{n}$ is a dividing cycle; if this is the case we shall call the exhaustion canonical.

2. Weak boundary component. Let $\gamma$ be an ideal boundary component of $F$, and let $\left\{F_{n}\right\}$ be a canonical exhaustion of $F$. Then there exists a component $\gamma_{n}$ of $\partial F_{n}$ which separates $\gamma$ from $F_{n}$. Let $n_{0}$ be a fixed number and consider the component $G_{n}$ of $\bar{F}_{n}-F_{n_{0}}\left(n>n_{0}\right)$ such that $\gamma_{n} \subset \partial G_{n}$. There exists a harmonic function $s_{n}(p)$ on $\overline{G_{n}}$ which satisfies the following conditions:

(i) $s_{n}=0$ on $\gamma_{n_{0}}$ and $\int_{\gamma_{n_{0}}} * d s_{n}=2 \pi,\left(\gamma_{n_{0}}=\partial F_{n_{0}} \cap \partial G_{n}\right)$

(ii) $s_{n}=\log r_{n}=$ const. on $\gamma_{n}$,

(iii) $s_{n}=$ const. on each component $\beta_{n \nu}$ of $\partial G_{n}-\gamma_{n}-\gamma_{n_{0}}$ and $\int_{\beta_{n \nu}} * d s_{n}=0$

The condition $\lim _{n \rightarrow \infty} r_{n}=\infty$ depends neither on $n_{0}$ nor on the exhaustion. If it is satisfied, $\gamma$ is said to be weak.

Weak boundary components were introduced for plane regions by Grötzch [1] in connection with the so-called Kreisnormierungsproblem. He called them vollkommen punktförmig. They were generalized for open Riemann surfaces by Sario [6] and discussed also by Savage [7] and Jurchescu [2]. The above definition was given by Jurchescu [2].

A noncompact subregion $N$ whose relative boundary $\partial N$ consists of a finite number of closed analytic curves is called a neighborhood of $\gamma$ if $\gamma$ is an ideal boundary component of $N$ as well. Let $\{c\}$ be the family of all cycles $c$ (i.e., unions of finite numbers of closed curves) which are in $N$ and separate $\gamma$ from $\partial N$. Jurchescu [2] showed that $\lambda\{c\}=0$ if and only if $\gamma$ is weak, where $\lambda\{c\}$ is the extremal length of the family $\{c\}$.

Received October 22, 1958. This paper was prepared under Contract No. DA-04-495ORD-722, OOR Project No. 1517 between the University of California, Los Angeles and the Office of Ordnance Research, U. S. Army. 
3. Savage's criterion. Let $\left\{F_{n}\right\}$ be an arbitrary exhaustion. Let $E_{n}$ be the smallest union of components of $F_{n}-\bar{F}_{n-1}$ such that $\gamma_{n-1}=$ $\partial E_{n} \cap \partial F_{n-1}$ is a cycle which separates $\gamma$ from $F_{n-1}(n=2,3, \cdots)$. Evidently $\gamma_{n} \subset \partial E_{n}$. If $\left\{F_{n}\right\}$ is canonical, $E_{n}$ is connected and $\gamma_{n}$ is a closed analytic curve.

There exists a harmonic function $u_{n}(p)$ on $\overline{E_{n}}$ such that

(i) $u_{n}=0$ on $\gamma_{n-1}$ and $\int_{\gamma_{n-1}} * d u_{n}=2 \pi$,

(ii) $u_{n}=\log \mu_{n}=$ const. on $\partial E_{n}-\gamma_{n-1}=\partial E_{n} \cap \partial F_{n}$.

The quantity $\log \mu_{n}$ is called the modulus of $E_{n}$ (cf. Sario [4,5], who called $\mu_{n}$ the modulus). It is expressed in terms of extremal length as follows :

$$
\log \mu_{n}=\frac{2 \pi}{\lambda\{c\}_{n}},
$$

where $\{c\}_{n}$ is the family of cycles in $E_{n}$ homologous to $\gamma_{n-1}$.

Since $\sum^{\infty} 1 / \lambda\{c\}_{n} \leqq 1 / \lambda\{c\}$, we get the following criterion:

THEOREM 1 (Savage [7]). If there exists an exhaustion such that $\prod_{n=2}^{\infty} \mu_{n}=\infty$, then $\gamma$ is weak.

The purpose of the present note is to discuss the converse of this theorem.

4. Jurchescu's criterion. Suppose the exhaustion $\left\{F_{n}\right\}$ is canonical. There exists a harmonic function $U_{n}(p)$ on $\bar{E}_{n}$ such that

(i) $U_{n}=0$ on $\gamma_{n-1}$ and $\int_{\gamma_{n-1}} * d U_{n}=2 \pi$,

(ii) $U_{n}=\log M_{n}=$ const. on $\gamma_{n}$,

(iii) $U_{n}=$ const. on each component $\beta_{n \nu}$ of $\partial E_{n}-\gamma_{n}-\gamma_{n-1}$ and $\int_{\beta_{n \nu}} * d U_{n}=0$.

Jurchesch's paper [2] contains implicitly the following result:

Theorem 2 (Jurchescu). A boundary component $\gamma$ is weak if and only if there exists a canonical exhaustion such that $\prod_{n=2}^{\infty} M_{n}=\infty$.

Proof. Sufficiency: Let $\left\{c^{\prime}\right\}_{n}$ be the family of cycles in $E_{n}$ separating $\gamma_{n}$ from $\gamma_{n-1}$. It is not difficult to see that $\log M_{n}=2 \pi / \lambda\left\{c^{\prime}\right\}_{n}$. Since $\sum^{\infty} 1 / \lambda\left\{c^{\prime}\right\}_{n} \leqq 1 / \lambda\{c\}$, we conclude that $\sum_{n=2}^{\infty} \log M_{n}=\infty$ implies $\lambda\{c\}=0$.

Necessity: Consider a canonical exhaustion $\left\{F_{n}^{0}\right\}$. The desired exhaustion $\left\{F_{n}\right\}$ is obtained by taking its subsequence as follows:

$F_{1}=F_{1}^{0}$. To define $F_{2}$, consider the quantity $r_{n}$ introduced in No. 2 with respect to $F_{n}^{0}-{\overline{F_{1}^{0}}}_{1}(n=2,3, \cdots)$. Take $n_{2}$ so large that $r_{n_{2}} \geqq 2$, 
and put $F_{2}=F_{n_{2}}^{0}$. Evidently $M_{2}=r_{n_{2}}$. Similarly, $F_{3}=F_{n_{3}}^{0}$ is defined by considering $F_{n}^{0}-\bar{F}_{n_{2}}^{0}\left(n=n_{2}+1, n_{2}+2, \cdots\right)$ and by taking $n_{3}>n_{2}$ so large that $r_{n_{3}} \geqq 2$ where $r_{n_{2}}$ is the quantity $r_{n}$ introduced in No. 2 with respect to $F_{n}^{0}-F_{n_{2}}^{\overline{0}}$. We have $M_{3}=r_{n_{3}}$. On continuing this process, we obtain a canonical exhaustion such that $\sum_{n=2}^{\infty} \log M_{n} \geqq \sum_{n=2}^{\infty} \log 2=\infty$. The idea of this proof was first used by Noshiro [3].

5. The converse of Savage's criterion. We shall now show that Savage's criterion in Theorem 1 is also necessary.

THEOREM 3. If $\gamma$ is weak, then there exists an exhaustion such that $\prod_{n=2}^{\infty} \mu_{n}=\infty$. It is not necessarily canonical.

Proof. By Theorem 2 there exists a canonical exhaustion $\left\{F_{n}^{0}\right\}$ such that $\prod_{n=2}^{\infty} M_{n}^{0}=\infty$. From this we construct a canonical exhaustion $\left\{F_{n}^{*}\right\}$ as follows :

$F_{1}^{*}=F_{1}^{0}$. To construct $F_{2}^{*}$, let $\partial E_{2}^{0}-\gamma_{1}^{0}-\gamma_{2}^{0}=\beta_{21} \cup \beta_{22} \cup \cdots \cup \beta_{2 k_{2}}$ be the decomposition into components, and let $H_{3}^{\nu}$ be the component of $F_{3}^{0}-F_{2}^{0}$ such that $\partial H_{3}^{\nu} \cap \bar{F}_{2}^{0}=\beta_{2 \nu}\left(\nu=1,2, \cdots, k_{2}\right) . \quad F_{2}^{*}$ is the union of $F_{1}^{*}, E_{2}^{0} \cup \gamma_{1}^{0}$, all the other components of $F_{2}^{0}-F_{1}^{0}$, and $\bigcup_{\nu=1}^{k_{2}} H_{3}^{\nu}$. In this way, $F_{n}^{*}$ is defined as the union of $F_{n-1}^{*}, E_{n}^{0} \cup \gamma_{n-1}^{0}$, every component of $F_{m+1}^{0}-F_{m}^{0}(m \geqq n)$ which is adjacent to $F_{n-1}^{*}$, and $\bigcup_{\nu=1}^{k_{n}} H_{n+1}^{\nu}$. By construction, $E_{n}^{*}=E_{n}^{0} \cup \bigcup_{\nu=1}^{k_{n}} H_{n+1}^{\nu}$.

The desired exhaustion $\left\{F_{n}\right\}$ is obtained by taking a refinement of $\left\{F_{n}^{*}\right\}$ as follows: Consider $E_{n}^{0}$ and the function $U_{n}^{0}$ for the exhaustion $\left\{F_{n}^{0}\right\}$. Let $\partial E_{n}^{0}-\gamma_{n}^{0}-\gamma_{n-1}^{0}=\beta_{n 1} \cup \beta_{n 2} \cup \cdots \cup \beta_{n k_{n}}$ be the decomposition into components and let $U_{n}^{0} \equiv a_{\nu}$ on $\beta_{n \nu}\left(\nu=1,2, \cdots, k_{n}\right)$. We may assume, without loss of generality, that the $a_{\nu}$ 's are different by pairs. We suppose that

$$
0 \equiv a_{0}<a_{1}<\cdots<a_{k_{n}}<a_{k_{n}+1} \equiv \log M_{n}^{0} .
$$

Take $a_{\nu}^{\prime}\left(a_{\nu-1}<a_{\nu}^{\prime}<a_{\nu} ; \nu=1,2, \cdots, k_{n}, a_{k_{n}+1}^{\prime} \equiv \log M_{n}^{0}\right)$ and $a_{\nu}^{\prime \prime}\left(a_{\nu}<a_{\nu}^{\prime \prime}<\right.$ $\left.a_{\nu+1} ; \nu=1, \cdots, k_{n}, a_{0}^{\prime \prime} \equiv 0\right)$ so close to $a_{\nu}$ that

$$
\sum_{\nu=1}^{k_{n}+1}\left(a_{\nu}^{\prime}-a_{\nu-1}^{\prime \prime}\right) \geqq \log M_{n}^{0}-2^{-n}
$$

Consider the sets

$$
\begin{aligned}
& D_{n}^{\nu}=\left\{p ; a_{\nu-1}^{\prime \prime}<U_{n}^{0}(p)<a_{\nu}^{\prime \prime}\right\}, \nu=1,2, \cdots, k_{n}+1,\left(a_{k_{n}+1}^{\prime \prime} \equiv \log M_{n}^{0}\right) \\
& D_{n}^{\prime \nu}=\left\{p ; a_{\nu-1}^{\prime \prime}<U_{n}^{0}(p)<a_{\nu}^{\prime}\right\}, \nu=1,2, \cdots, k_{n}+1 .
\end{aligned}
$$

The modulus $\log \mu^{\prime(\nu)}$ of $D_{n}^{\prime \nu}$ with respect to $\beta^{\nu}=\left\{p ; U_{n}^{0}(p)=a_{\nu-1}^{\prime \prime}\right\}$ and $\partial D_{n}^{\prime \nu}-\beta^{\prime}$ is equal to $a_{\nu}^{\prime}-a_{\nu-1}^{\prime \prime}$, since the function $U_{n}^{0}(p)-a_{\nu-1}^{\prime \prime}$ plays the role of $u_{n}(p)$ introduced in No. 3. Let $\log \mu^{(\nu)}$ be the modulus of $D_{n}^{\nu}$ 
with respect to $\beta^{\nu}$ and $\partial D_{n}^{\nu}-\beta^{\nu}$. Since $\mu^{(\nu)} \geqq \mu^{\prime(\nu)}$, we obtain, by (1),

$$
\sum_{\nu=1}^{k_{n}+1} \log \mu^{(\nu)} \geqq \log M_{n}^{0}-2^{-n} .
$$

We have decomposed $E_{n}^{0}$ into $k_{n}+1$ subsets $D_{n}^{\nu}$. $E_{n}^{*}-E_{n}^{0}$ consists of components $H_{n+1}^{\nu}$ such that $\beta_{n \nu}=\partial H_{n+1}^{\nu} \cap \partial E_{n}^{0}\left(\nu=1,2, \cdots, k_{n}\right)$. By decomposing $H_{n+1}^{\nu}$ into $k_{n}-\nu+1$ slices, we obtain a decomposition of $E_{n}^{*}$ into $k_{n}+1$ parts. It is possible to divide each of the other components of $F_{n}^{*}-\bar{F}_{n-1}^{*}$ into $k_{n}+1$ pieces so that we get an exhaustion $\left\{F_{n}\right\}$ which is a refinement of $\left\{F_{n}^{*}\right\} . D_{n}^{\nu}$ plays the role of $E_{n}$ with respect to this exhaustion. Therefore, by (2), we get

$$
\sum_{n=2}^{\infty} \log \mu_{n} \geqq \sum_{n=2}^{\infty} \log M_{n}^{0}-1=\infty \text {. }
$$

6. Remark. On a "schlichtartig" surface, every exhaustion is canonical. If $F$ is an arbitrary Riemann surface, the question arises whether or not Savage's criterion is still necessary under the restriction that $\left\{F_{n}\right\}$ is canonical. The answer is given by

THEOREM 4. There exist a $\gamma$ of an $F$ which is weak and such that $\Pi_{n=2}^{\infty} \mu_{n}<\infty$ for every canonical exhaustion.

Construction of $F$ : In the plane $|z|<\infty$, consider the closed intervals

$$
I_{k}:\left[2^{k^{2}}, 2^{k^{2}}+1\right] \quad(k=2,3, \cdots)
$$

on the positive real axis, and the circular arcs

$$
\begin{gathered}
\alpha_{\nu}:|z|=\nu,|\arg z| \leqq \frac{\pi}{2} \\
\left(\nu=2^{k_{2}}+2,2^{k^{2}}+3, \cdots, 2^{(k+1)^{2}}-1 ; k=2,3, \cdots\right) .
\end{gathered}
$$

Take two replicas of the slit plane $(|z|<\infty)-\bigcup_{k=2}^{\infty} I_{k}$ and connect them crosswise across $I_{k}(k=2,3, \cdots)$. From the resulting surface, delete all the $\alpha_{\nu}$ 's on both sheets. This is a Riemann surface $F$ of infinite genus.

$F$ has an ideal boundary component $\gamma$ over $z=\infty$, which is evidently weak.

Let $\left\{F_{n}\right\}$ be an arbitrary canonical exhaustion. Consider $E_{n}$ corresponding to $\gamma$ (No. 3). The interval $I_{k}$ determines a closed analytic curve $C_{k}$ on $F$. Since $\gamma_{n-1}=\partial E_{n} \cap \bar{F}_{n-1}$ is a dividing cycle, the intersection number $\gamma_{n-1} \times C_{k}$ vanishes and, therefore, $\gamma_{n-1} \cap C_{k}$ consists of an even number of points whenever it is not void.* Take two consecutive points

* Added in proof. We should have mentioned the case where $\gamma_{n-1}$ tangents $C_{k}$. The following discussion covers this case if the number of the points of $\gamma_{n-1} \cap C_{k}$ is counted with the multiplicity of tangency and case $p=q$ is not excluded. 
$p$ and $q$ in $\gamma_{n-1} \cap C_{k}$. There are two possibilities according as the arc $\overparen{p q} \subset \gamma_{n-1}$ is homotopic to $\overparen{p q} \subset C_{k}$ or not. If the latter case happens for at least one pair of $p$ and $q$, we shall say that $\gamma_{n-1}$ intersects $C_{k}$ properly.

Since $\gamma_{n-1}$ is a closed curve separating $\gamma$ from $F_{n-1}$, there exists a number $k$ such that $\gamma_{n-1}$ intersects $C_{k}$ properly. If there is more than one $k$, we take the greatest one and denote it by $k(n)$.

To estimate $\mu_{n}$, let $\{c\}_{n}$ be the family of all cycles in $E_{n}$ separating $\gamma_{n-1}$ from $\partial E_{n}-\gamma_{n-1}$. We have mentioned that $\log \mu_{n}=2 \pi / \lambda\{c\}_{n}$. Let $C_{k}$ be a curve for which there are numbers $n$ with $k(n)=k$. Evidently these $n$ are finite in number and consecutive. Let $n_{k}$ be the greatest.

I. If $k(n)=k$ and $n<n_{k}$ then $\gamma_{n-1}$ and $\gamma_{n}$ intersect $C_{k}$ properly. Since every $c \in\{c\}_{n}$ separates $\gamma_{n-1}$ from $\gamma_{n}$, it has a component which intersects $C_{k}$ and is not completely contained in the doubly connected region $\Delta_{k}$ consisting of all points that lie over $\left\{z ; 2^{k^{2}}-1<|z|<2^{k^{2}}+\right.$ $2,|\arg z|<\pi / 2\}$. Therefore, every $c$ contains a curve in $\left\{c^{\prime}\right\}^{(k)}$ which is the family of all curves in the right half-plane connecting $I_{k}$ with the imaginary axis. Consequently

$$
\sum_{\substack{k(n)=k \\ n \neq n_{k}}} \frac{1}{\lambda\{c\}_{n}} \leqq \frac{1}{\lambda\left\{c^{\prime}\right\}^{(k)}} .
$$

II. $k(n)=k$ and $n=n_{k}$. Consider all the $\alpha_{\nu}\left(\nu \geqq 2^{k^{2}}+2\right)$ on the upper sheet. Let $G_{n-1}$ be the component of $F-\bar{F}_{n-1}$ such that $\partial G_{n-1}=$ $\gamma_{n-1}$. For a sufficiently large $\nu, \alpha_{\nu}$ is an ideal boundary component of $G_{n-1}$. Let $\nu(k)$ be the least $\nu$ with this property. If $\nu(k)=2^{k^{2}}+2$, then every $c \in\{c\}_{n}$ separates $\gamma_{n-1}$ from $\alpha_{\nu(k)}$ and, therefore, it has a component intersects either $C_{k}$ or one of four line segments over $\left[2^{k^{2}}-1,2^{k^{2}}\right]$ or $\left[2^{k^{2}}+1,2^{k^{2}}+2\right]$. When $\nu(k)=2^{2^{2}}+2$ for some $l>k$, then $\gamma_{n-1}$ separates $\alpha_{\nu(k)-3}$ from $\alpha_{\nu(k)}$ and every $c \in\{c\}_{n}$ separates $\gamma_{n-1}$ from $\alpha_{\nu(k)}$, so that $c$ has a component with the above property. If $\nu(k)$ is not of the form $2^{2^{2}}+2$, then, for the same reason, every $c \in\{c\}_{n}$ has a component which intersects the line segment on the upper sheet lying over $[\nu(k)-1, \nu(k)]$, and is not contained in the simply connected region on the upper sheet consisting of all points over $\{z ; \nu(k)-1<|z|<\nu(k)$, $|\arg z|<\pi / 2\}$. In any case, every $c \in\{c\}_{n}$ contains a curve in $\left\{c^{\prime \prime}\right\}^{(k)}$ which is the family of all curves in the right half-plane connecting $[\nu(k)-3, \nu(k)]$ with the imaginary axis. Therefore,

$$
\frac{1}{\lambda\{c\}_{n}} \leqq \frac{1}{\lambda\left\{c^{\prime \prime}\right\}^{(k)}}
$$

By (3) and (4), we obtain

$$
\sum_{n=2}^{\infty} \log \mu_{n}=2 \pi \sum_{n=2}^{\infty} \frac{1}{\lambda\{c\}_{n}} \leqq 2 \pi \sum_{k=2}^{\infty}\left(\frac{1}{\lambda\left\{c^{\prime}\right\}^{(k)}}+\frac{1}{\lambda\left\{c^{\prime \prime}\right\}^{(k)}}\right) .
$$


To show the convergence of $\sum_{k=2}^{\infty} 1 / \lambda\left\{c^{\prime}\right\}^{(k)}$, we make use of the transformation $z \rightarrow z^{2}$. It is immediately seen that $\lambda\left\{c^{\prime}\right\}^{(k)}$ is equal to the extremal distance between $[-\infty, 0]$ and $I_{k}^{\prime}=\left[2^{2 k^{2}},\left(2^{k^{2}}+1\right)^{2}\right]$ with respect to the region $A=\left\{[-\infty, 0] \cup I_{k}^{\prime}\right\}^{c}$. Since $A$ is conformally equivalent to Teichmüller's extremal region $\{[-1,0] \cup[P, \infty]\}^{c}$ where

$$
P=\frac{2^{2 k^{2}}}{\left(2^{k^{2}}+1\right)^{2}-2^{2 k^{2}}},
$$

we have (Teichmüller [8])

$$
\begin{aligned}
\lambda\left\{c^{\prime}\right\}^{(k)} & \sim \frac{\log P}{2 \pi} \quad(P \rightarrow \infty) \\
& \sim \frac{k^{2} \log 2}{2 \pi} \quad(k \rightarrow \infty),
\end{aligned}
$$

and, therefore, $\sum_{k=2}^{\infty} 1 / \lambda\left\{c^{\prime}\right\}^{(k)}<\infty$. Similarly $\sum_{k=2}^{\infty} 1 / \lambda\left\{c^{\prime \prime}\right\}^{(k)}<\infty$ because $\nu(k) \geqq 2^{k^{2}}+2$. We conclude that

$$
\sum_{n=2}^{\infty} \log \mu_{n}<\infty
$$

\section{REFERENCES}

1. H. Grötzsch, Eine Bemerkung zum Koebeschen Kreisnormierungsprinzip, Ber. Verh. Sächs. Akad. Wiss. Leipzig. Math.-Nat. Kl., 87 (1935), 319-324.

2. M. Jurchescu, Modulus of a boundary component, Pacific J. Math., 8 (1958), 791-809.

3. K. Noshiro, Open Riemann surface with null-boundary, Nagoya Math. J., 3 (1951), 73-79.

4. L. Sario, Über Riemannsche Flächen mit hebbarem Rand, Ann. Acad. Sci. Fenn. Ser. A. I, 50 (1948), pp. 79.

5. —_, Modular criteria on Rieman surfaces, Duke Math. J., 20 (1953), 279-286.

6. - Capacity of the boundary and of a boundary component, Ann. Math., 59 (1954), 135-144.

7. N. Savage, Weak boundary components of an open Riemann surface, Duke Math. J., 24 (1957), 79-96.

8. O. Teichmüller, Untersuchungen über konforme und quasikonforme Abbibldung, Deutsche Math., 3 (1938), 621-678.

University of CALIfornia, Los ANgeles, AND

TOKYo Institute of TEChNOLOGY 


\section{PACIFIC JOURNAL OF MATHEMATICS}

EDITORS

David Gilbarg

Stanford University

Stanford, California

R. A. Beaumont

University of Washington

Seattle 5 , Washington
A. L. Whiteman

University of Southern California

Los Angeles 7, California

L. J. PAIGE

University of California

Los Angeles 24, California

\section{ASSOCIATE EDITORS}

\author{
E. F. BECKENBACH \\ C. E. BURGESS \\ E. HEWITT \\ A. HORN
}

\author{
V. GANAPATHY IYER \\ R. D. JAMES \\ M. S. KNEBELMAN \\ L. NACHBIN
}
I. NIVEN
E. G. STRAUS
T. G. OSTROM
G. SZEKERES
H. L. ROYDEN
F. WOLF
M. M. SCHIFFER
K. YOSIDA

\section{SUPPORTING INSTITUTIONS}

\author{
UNIVERSITY OF BRITISH COLUMBIA \\ CALIFORNIA INSTITUTE OF TECHNOLOGY \\ UNIVERSITY OF CALIFORNIA \\ MONTANA STATE UNIVERSITY \\ UNIVERSITY OF NEVADA \\ OREGON STATE COLLEGE \\ UNIVERSITY OF OREGON \\ OSAKA UNIVERSITY \\ UNIVERSITY OF SOUTHERN CALIFORNIA
}

\author{
STANFORD UNIVERSITY \\ UNIVERSITY OF TOKYO \\ UNIVERSITY OF UTAH \\ WASHINGTON STATE COLLEGE \\ UNIVERSITY OF WASHINGTON \\ $* \quad * \quad *$ \\ AMERICAN MATHEMATICAL SOCIETY \\ CALIFORNIA RESEARCH CORPORATION \\ HUGHES AIRCRAFT COMPANY \\ SPACE TECHNOLOGY LABORATORIES
}

Mathematical papers intended for publication in the Pacific Journal of Mathematics should be typewritten (double spaced), and the author should keep a complete copy. Manuscripts may be sent to any one of the four editors. All other communications to the editors should be addressed to the managing editor, L. J. Paige at the University of California, Los Angeles 24, California.

50 reprints per author of each article are furnished free of charge; additional copies may be obtained at cost in multiples of 50 .

The Pacific Journal of Mathematics is published quarterly, in March, June, September, and December. The price per volume (4 numbers) is $\$ 12.00$; single issues, $\$ 3.50$. Back numbers are available. Special price to individual faculty members of supporting institutions and to individual members of the American Mathematical Society: $\$ 4.00$ per volume; single issues, $\$ 1.25$.

Subscriptions, orders for back numbers, and changes of address should be sent to Pacific Journal of Mathematics, 2120 Oxford Street, Berkeley 4, California.

Printed at Kokusai Bunken Insatsusha (International Academic Printing Co., Ltd.), No. 6, 2-chome, Fujimi-cho, Chiyoda-ku, Tokyo, Japan.

PUBLISHED BY PACIFIC JOURNAL OF MATHEMATICS, A NON-PROFIT CORPORATION

The Supporting Institutions listed above contribute to the cost of publication of this Journal, but they are not owners or publishers and have no responsibility for its content or policies. 


\section{Pacific Journal of Mathematics}

\section{Vol. 9, No. 4 \\ August, 1959}

Frank Herbert Brownell, III, A note on Kato's uniqueness criterion for

Schrödinger operator self-adjoint extensions ............... 953

Edmond Darrell Cashwell and C. J. Everett, The ring of number-theoretic

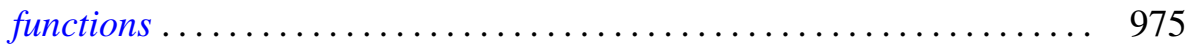

Heinz Otto Cordes, On continuation of boundary values for partial

differential operators ............................. 987

Philip C. Curtis, Jr., n-parameter families and best approximation . . . . . . 1013

Uri Fixman, Problems in spectral operators . . . . . . . . . . . . . . . 1029

I. S. Gál, Uniformizable spaces with a unique structure .............. 1053

John Mitchell Gary, Higher dimensional cyclic elements ............ 1061

Richard P. Gosselin, On Diophantine approximation and trigonometric

polynomials ..................................... 1071

Gilbert Helmberg, Generating sets of elements in compact groups ........ 1083

Daniel R. Hughes and John Griggs Thompson, The H-problem and the

structure of $H$-groups .................................. 1097

James Patrick Jans, Projective injective modules ................. 1103

Samuel Karlin and James L. McGregor, Coincidence properties of birth and

death processes ..................................... 1109

Samuel Karlin and James L. McGregor, Coincidence probabilities ........ 1141

J. L. Kelley, Measures on Boolean algebras ................... 1165

John G. Kemeny, Generalized random variables ................... 1179

Donald G. Malm, Concerning the cohomology ring of a sphere bundle ... . . 1191

Marvin David Marcus and Benjamin Nelson Moyls, Transformations on

tensor product spaces .................................. 1215

Charles Alan McCarthy, The nilpotent part of a spectral operator ........ 1223

Kotaro Oikawa, On a criterion for the weakness of an ideal boundary

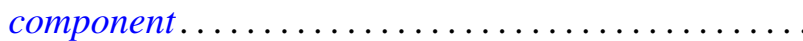

Barrett O'Neill, An algebraic criterion for immersion . . ............... 1239

Murray Harold Protter, Vibration of a nonhomogeneous membrane ... . . . . 1249

Victor Lenard Shapiro, Intrinsic operators in three-space . . . . . . . . . . . 1257

Morgan Ward, Tests for primality based on Sylvester's cyclotomic

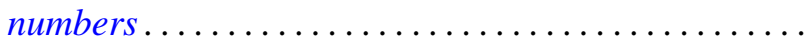

L. E. Ward, A fixed point theorem for chained spaces ....

Alfred B. Willcox, Šilov type $C$ algebras over a connected locally compact

abelian group..................................... 1279

Jacob Feldman, Correction to "Equivalence and perpendicularity of

Gaussian processes" ........................ 